\title{
Intermédialités
}

Histoire et théorie des arts, des lettres et des techniques

Intermediality

History and Theory of the Arts, Literature and Technologies

\section{L’expérience et son double}

\section{Notes sur la reconstruction d'expositions et la photographie}

\section{Elitza Dulguerova}

Numéro 15, printemps 2010

exposer

displaying

URI : https://id.erudit.org/iderudit/044674ar

Aller au sommaire du numéro

Éditeur(s)

Revue Intermédialités (Presses de l’Université de Montréal)

ISSN

1705-8546 (imprimé)

1920-3136 (numérique)

Découvrir la revue

Citer cet article

Dulguerova, E. (2010). L'expérience et son double : notes sur la reconstruction d'expositions et la photographie. Intermédialités / Intermediality, (15), 53-71.
Résumé de l'article

La reconstitution d'expositions passées est devenue, depuis une trentaine d'années, une stratégie réflexive critique adoptée par plusieurs musées et, parfois, par certains artistes d'allégeance néoconceptuelle. En partant de l'hypothèse que dans de tels cas la réplique porte moins sur l'objet lui-même que sur l'expérience de cet objet, ce texte s'intéresse à l'oscillation qui en résulte : entre le désir de regagner prise sur le passé en écourtant la distance temporelle et celui de riposter depuis le présent en creusant l'écart de l'impossible rencontre. Il interroge en particulier le rôle de la photographie comme élément de référence de telles répliques à travers des exemples de reconstructions partielles réalisées à la fois par des institutions muséales (l'exposition The Avant-Garde in Russia : New Perspectives 1900-1930, Los Angeles County Museum of Art, 1980) et par des artistes (Simon Starling, Nachbau, Museum Folkwang, Essen, 2007). 


\title{
L'expérience et son double
}

\section{Notes sur la reconstruction d'expositions et la photographie}

\author{
Elitza Dulguerova
}

a première illustration de ce texte présente une reconstruction délibérément citationnelle, et tout aussi délibérément imparfaite, d'une manifestation avant-gardiste tenue en 1915 à Petrograd, La dernière exposition futuriste de tableaux o,10 (zéro-dix) ${ }^{1}$ (fig. 1). Un blow-up de la photographie emblématique de l'exposition source est exposé à côté de l'accrochage contemporain dont le manque de conformité à l'égard de l'«original» nous assure qu'il ne s'agit pas de la même exposition, tout en évoquant de façon indicielle sa mémoire. Cette mise en scène des œuvres suprématistes de Kazimir Malevitch fut présentée - et photographiée - lors de l'exposition The Avant-Garde in Russia : New Perspectives 1910-1930 réalisée au Los Angeles County Museum of Art (LACMA) en 1980.

Trente ans plus tard, dans le catalogue de l'exposition rétrospective Vides du Centre Pompidou à Paris en 2009, l'un des commissaires définissait en ces termes le défi que devaient relever les pratiques muséales contemporaines:

En pleine queue de comète d'un certain post-modernisme, Vides est aussi une contribution au débat sur la répétition et la reproductibilité de l'exposition qui est enfin devenue un objet d'analyse de l'histoire de l'art. Depuis notamment Stationen der Moderne en 1988 à Berlin, l'exposition rétrospective d'expositions est née. [...] Entre le «j’y étais» fragmentaire et les archives rarement conservées, le partiel et le partial sont le lot commun du souvenir d'exposition².

1. La dernière exposition futuriste de tableaux o,1o (zéro-dix) avait réuni quatorze artistes à Petrograd du 19 décembre 1915 au 19 janvier 1916 dans le Bureau artistique de Natalia Dobytchina. Son catalogue est reproduit en fac-similé et traduit en français dans Herman Berninger et Jean-Albert Cartier, Pougny. Jean Pougny (Iwan Puni) 1892-1956. Catalogue de l'œuvre, vol. 1 : Les années d'avant-garde, Russie, Berlin, 1910-1923, Tübingen, Ernst Wasmuth, 1972, p. 58-59 et 157-158.

2. Laurent Le Bon, "Qui ne risque rien n'a rien», dans John Armleder, Mathieu Copeland, Laurent Le Bon, Gustav Metzger, Mai-Thu Perret, Clive Philippot et Philippe Pirotte (dir.), Vides : une rétrospective, Paris, Éditions du Centre Pompidou, 2009, p. 164-165. 
Ce constat témoigne en effet de l'adoption par plusieurs musées d'art contemporain d'une attitude autoréflexive aux apparences critiques qui se manifeste soit par des reconstitutions partielles de situations d'exposition au sein de manifestations plus grandes qu'elles permettent ainsi de contextualiser, soit par la mise en place d'expositions ayant les expositions pour sujet ${ }^{3}$. Cette approche de l'exposition comme donnée «reproductible», et de l'histoire des œuvres par le biais de leur contexte de présentation, prend en effet son essor dans les années 1980. La grande rétrospective Stationen der Moderne (Berlinische Galerie, 1988) qui revenait sur vingt expositions importantes pour l'histoire de l'Allemagne moderne, depuis Die Brücke jusqu'à la galerie télévisuelle de Gerry Schum des années 1970, est souvent citée comme la première occurrence de ces pratiques

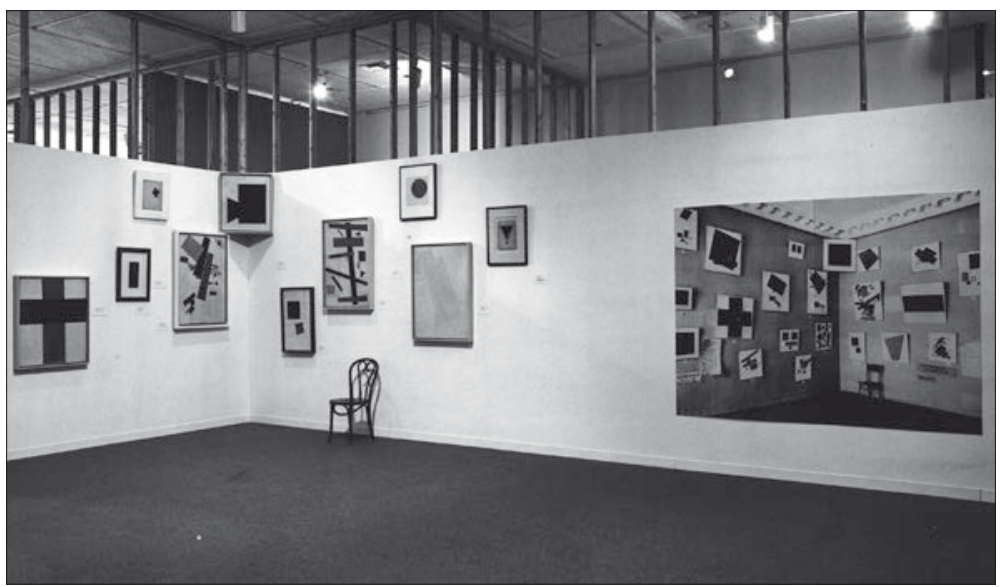

Fig. 1: Vue partielle de l'accrochage de l'exposition The Avant-Garde in Russia: New Perspectives 19101930, Los Angeles County Museum of Art, 8 juillet-28 septembre 1980. Commissaires d'exposition: Stephanie Barron et Maurice Tuchman. Architecture intérieure: Frank Gehry. (C) Archive photographique du LACMA.

3. L'exemple le plus récent revient au Van Abbemuseum d'Eindhoven sous la direction de Charles Esche. Les différents volets de son programme biennal Play Van Abbe (2009-2011) abordent des «questions topiques sur l'identité et les fonctions des musées en général» en exposant non seulement les œuvres mais surtout «la façon dont nous sommes autorisés à les regarder et à en parler au sein d'un musée » : manuel Play Van Abbe-Part 2, Eindhoven, 2010, p. 24 (ma traduction). L'un des volets, Time Machines (avrilseptembre 2010), cible ces problématiques précisément par la reconstitution - partielle et partiale - d'expositions passées. Voir également Martha Buskirk, «Play Van Abbe», Artforum, vol. XLVIII, n ${ }^{\circ} 10$ "The Museum Revisited », été 2010, p. 342-343.

4. Michael Bollé et Eva Züchner (dir.), Stationen der Moderne: die bedeutenden Kunstausstellungen des 20. Jahrhunderts in Deutschland, Berlin, Berlinische Galerie et Nikolai, 1988. 
Mais, comme nous le verrons, la tendance était déjà en marche depuis au moins une dizaine d'années. Simultanément à cet intérêt des musées pour le contexte d'énonciation des œuvres, le monde académique a vu l'émergence d'un nouveau sous-genre d'histoire de l'art - l'histoire des expositions - et de qualificatifs tels que Ausstellungskünstler (artiste d'exposition) 5 .

Il convient donc de réfléchir à cette tendance de plus en plus prégnante, au sein des musées d'art, à répliquer des expositions : non seulement à reconstruire des œuvres disparues, mais à reconstituer des situations de présentation révolues. De telles reconstitutions nous informent soit du moment de la première émergence des œuvres (expositions initiales), soit du contexte dans lequel elles ont évolué auparavant (ateliers, lieux de commande, précédent accrochage...). Elles répliquent moins un objet donné que l'expérience de cet objet. Or, ce raisonnement conduit à une série de paradoxes riegliens, si ce n’est aux limites du musée dans son rapport à l'histoire et à la mémoire: quel serait le point de départ à partir duquel on peut recréer une expérience révolue? Peut-on répliquer l'expérience passée d'une œuvre? S'agit-il plutôt de répliquer à l'expérience passée de l'œuvre, à partir du moment présent? Mais dans ce cas, ne fait-on pas l'impasse du passé?

Ce texte se propose de chercher des éléments de réponse à ces questions dans l'étude des moyens (médias) par lesquels s'opèrent ces reconstitutions, et en particulier de la relation entre reconstruction spatiale et photographie. Les exemples analysés sont issus de pratiques muséales, comme pour le LACMA en 1980 et de la Galerie Tretiakov de Moscou en 2006 et, dans un deuxième temps, de la collaboration entre artiste et musée, comme dans le projet Nachbau réalisé par Simon Starling pour le Museum Folkwang d'Essen en 2007.

L'historienne de l'art Reesa Greenberg a récemment proposé une typologie de ces nouvelles postures d'exposition. L'intitulé anglais de son article,

5. Voir par exemple Katharina Hegewisch et Bernd Klüser (dir.), L'art de l'exposition. Une documentation sur trente expositions exemplaires du $20^{e}$ siècle [1991], trad. Denis Trierweiler, Paris, Les Éditions du Regard, 1998; Bruce Altshuler, The Avant-Garde in Exhibition. New Art in the $20^{\text {th }}$ Century, New York, Harry N. Abrams Inc. Publishers, 1994; Bruce Altshuler and Phaidon editors (dir.), Salon to Biennial. Exhibitions That Made Art History, vol. 1: 1863-1959, Londres et New York, Phaidon, 2008; Jérôme Glicenstein, L'art, une histoire d'expositions, Paris, Les Presses universitaires de France, coll. «Lignes d'art», 2009; Oskar Bätschmann, The Artist in the Modern World. The Conflict between Market and Self-Expression, Cologne, DuMont Buchverlag, 1997. 
«Remembering Exhibitions ${ }^{6}$ », offre un vacillement productif entre mode transitif et réflexif, entre sujet et objet. Pour penser ce travail de commémoration, Reesa Greenberg suggère trois catégories: replica, reconstitutions partielles d'expositions passées, souvent bien documentées, dans un but de célébration, d'hommage ou de recherche; riff, expositions dans lesquelles le travail de mémoire va de pair avec l'oubli, de sorte qu'il s'agit davantage de variations et de détournements que de répliques; enfin, reprise, terme qui désigne la façon dont les expositions construisent leur propre mémoire (catalogues, sites Web, etc.).

Les concepts de travail que j'avais formulés en réfléchissant à ces problématiques recoupent en partie ceux de Greenberg tout en visant un ensemble de cas plus spécifiques. Le concept de «réplique» sera ici décliné en deux souscatégories, la reprise et la riposte, qui rejoignent, grosso modo, la distinction de Greenberg entre replica et riff. Lusage fait ici du terme «réplique » se rapproche de sa définition usuelle, même si l'approbation et la participation de l'inventeur, qui constituent une condition nécessaire, ne sont pas un facteur déterminant dans les cas étudiés: «[elle] implique la copie exacte du sujet, mais diffère de la répétition car la matière peut changer, de même que la dimension par rapport à l'original ${ }^{7}$ ». De plus, étant donné qu'il s'agit de répliques d'expositions et non, comme de coutume, de tableaux ou d'œuvres à support unique, le terme «réplique » peut être aussi entendu dans un sens proche de «reconstruction », traditionnellement réservé à l'architecture ${ }^{8}$.

\section{RÉPLIQUES : REPRISES, RIPOSTES}

La réplique peut parfois pencher vers la reprise, soit la répétition plus ou moins fidèle et historiquement exhaustive d'une situation d'exposition antérieure. Ainsi, la Galerie nationale Tretiakov de Moscou reconstituait en 2006, dans les salles de sa collection permanente, une exposition collective de la Société des Jeunes Artistes (Obmokhou), tenue en mai-juin 1921 à Moscou (fig. 2). En 1921, cette

6. Reesa Greenberg, «Remembering Exhibitions: From Point to Line to Web», Tate Papers, $\mathrm{n}^{\circ}$ 12, «Landmark Exhibitions», automne 2009, disponible à: www.tate.org. uk/research/tateresearch/tatepapers/ogautumn/greenberg.shtm (dernière consultation le 20 juillet 2010). Je tiens à exprimer ma gratitude à l'égard de Reesa Greenberg pour les stimulantes discussions que nous avons menées au fil des dernières années sur ces sujets d'intérêt mutuel.

7. Jacques Girard, Dictionnaire des termes d'art et d'archéologie, Paris, Klincksieck, 2007, p. 214.

8. Jean-Pierre Néraudau, Dictionnaire d'histoire de l'art, préface de Pierre Grimal, Paris, Les Presses universitaires de France, 1985, p. 406. 


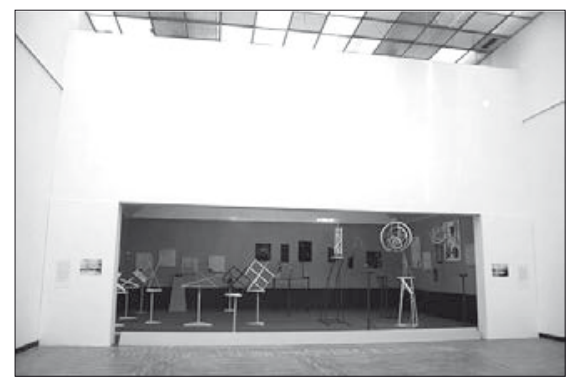

Fig. 2: Reconstruction de l'exposition de l'Obmokhou de 1921 dans les salles de la collection permanente de la Galerie nationale Tretiakov, Moscou. Auteur: Viatcheslav Koléïtchouk, 2006. Exécution SARL «Vkhoutemas XXI" siècle». (C) Fonds «Novyi », Moscou.

exposition avait permis aux constructivistes dits «de laboratoire » de présenter publiquement les résultats des débats menés pendant les quatre mois précédents entre artistes, architectes et historiens de l'art au sein de l'Institut de culture artistique de Moscou (Inkhouk) sur la définition du concept de «construction» comme futur fondement de l'art' (fig. 3).

La reprise de 2006 fut effectuée par l'artiste Viatcheslav Koléïtchouk à l'initiative de la Galerie Tretiakov dans le cadre de la réorganisation de la présentation de sa collection. La réplique «à l'identique» ne s'applique pas uniquement aux œuvres - constructions spatiales expérimentales, fragiles et désormais inexistantes, d’Alexandre Rodtchenko, Karl Ioganson, Konstantin Medounetski, des frères Guéorgui et Vladimir Stenberg -, mais également à leur accrochage et disposition spatiale, à la dimension de la salle et jusqu'aux structures architecturales du plafond. Bien entendu, cette reprise ne se dissimule pas en tant que telle : l'effet théâtral évident est assumé par cette trouée dans le mur du musée d'une "scène » encadrée de part et d'autre par des textes explicatifs et des vues photographiques d'époque. Le communiqué de presse désigne explicitement cette Reconstruction de l'exposition de l'Obmokhou de 1921 comme «installation» et explique que celle-ci «est introduite au sein de l'exposition permanente comme exemple du premier constructivisme, dit "de laboratoire" (auteur: V. F. Koléitchouk) $)^{10} »$.

9. Voir Maria Gough, The Artist as Producer. Russian Constructivism in Revolution, Berkeley, University of California Press, 2005, p. 61-99.

10. Le communiqué de presse du 15 novembre 2007 mentionne ce projet comme un des éléments de la réorganisation de l'accrochage des salles d'art contemporain de la galerie Tretiakov et précise que le projet a bénéficié du soutien du Fonds «Le nouveau », alors que l'exécution du design conçu par Viatcheslav Koleitchouk a été confiée à la 


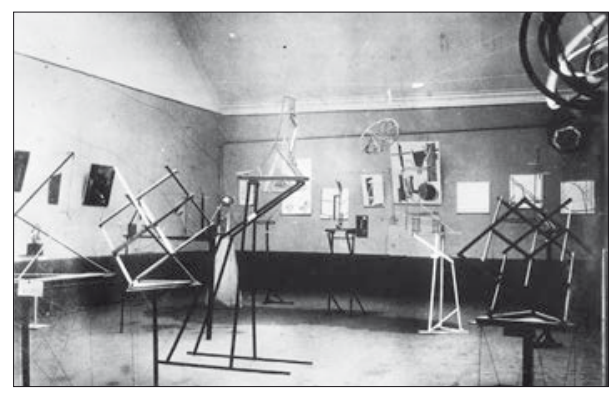

Fig. 3: Deuxième exposition de la Société des jeunes artistes (Obmokhou), Moscou, mai-juin 1921. Constructions spatiales de K. Ioganson, A. Rodtchenko, V. et G. Stenberg, K. Medounetski. Au mur: dessins et tableaux de V. Stepanova, A. Rodtchenko. Gracieuseté des Archives Alexandre Rodtchenko et Varvara Stepanova, Moscou.

Au-delà de l'objectif didactique indéniable, cette reconstruction très minutieuse produit l'illusion qu'une capsule temporelle du passé se trouve désormais à portée de main. Or, en même temps, et malgré sa volonté de fidélité historique, elle trahit son «original» en transformant l'exposition collective de 1921 en œuvre d'art unique, figeant la recherche «de laboratoire» en une «installation» avant la lettre dont les éléments constitutifs ne peuvent désormais plus être déplacés. Et même si les photographies et les textes informent le spectateur de la distance temporelle qui sépare 2006 de 1921, la réplique elle-même, dans sa perfection immuable, supprime cet écart en créant l'illusion d'un transfert dans le temps d'un passé fixe, encapsulé et non changé depuis 1921 dont seul le seuil de la salle d'exposition nous séparerait.

Vingt-cinq ans plus tôt, l'exposition The Avant-Garde in Russia: New Perspectives 1910-1930 était parmi les premières à intégrer au sein du muséecette dimension autoréflexive, en reconnaissant que la mémoire des accrochages faisait partie intégrale de l'histoire des œuvres et de l'expérience que les spectateurs pouvaient en faire.

L'exposition de l'Obmokhou de 1921 était l'un des éléments repris dans le show du LACMA, selon des modalités différentes de celles analysées dans l'exemple de la Galerie Tretiakov (fig. 4). Ce deuxième sens de la réplique n'était plus transitif (répliquer quelque chose, le dupliquer), mais intransitif (répliquer à, que le Petit Robert définit comme une «réponse vive faite avec humour»). Autrement dit, plutôt que la reprise, la riposte.

société à responsabilité limitée «Vkhoutemas $21^{\mathrm{e}}$ siècle ». Voir www.tretyakovgallery.ru/ru/ museum/news $/ 589 /$ (dernière consultation le 20 juillet 2010). 


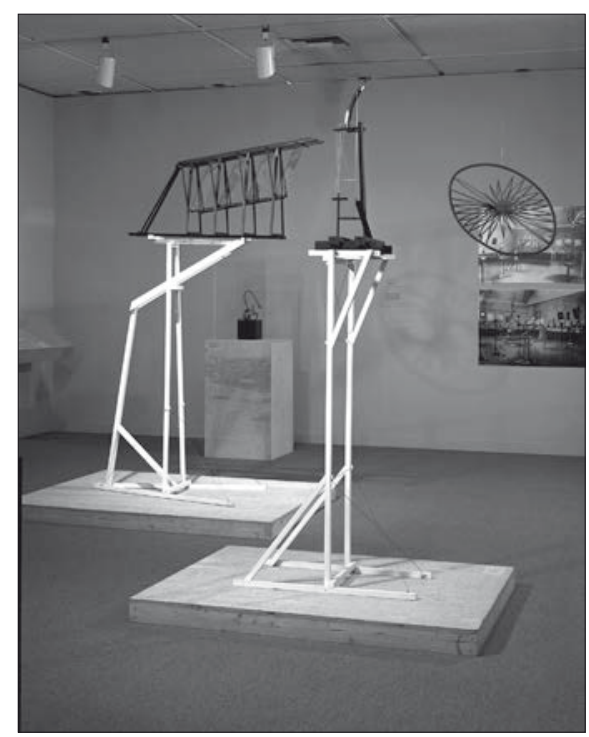

Fig. 4: Vue partielle de l'accrochage de l'exposition The Avant-Garde in Russia: New Perspectives 19101930, Los Angeles County Museum of Art, 8 juillet-28 septembre 1980. Reconstructions de pièces de G. Stenberg, A. Rodtchenko, K. Medounetski provenant de la $2^{\mathrm{e}}$ exposition d'Obmokhou. Au mur: photographies de la $2^{\mathrm{e}}$ exposition d'Obmokhou. (C) Archive photographique du LACMA.

Au lieu de reconstituer systématiquement toutes les constructions exposées en 1921 et de reproduire l'agencement spatial visible sur les photographies d'époque, le LACMA n'avait inclu qu'un nombre limité de reconstructions et les avait présentées sur des socles proéminents ou sous vitrine, indiquant ainsi sans ambages leur encadrement par l'institution muséale. Tout comme pour la réplique de l'exposition o,10, deux vues photographiques de l'exposition de 1921 jouxtaient les reconstructions, amenant le visiteur à faire, littéralement, le vaet-vient entre la représentation d'un événement passé dans la photographie, et l'évocation contemporaine de cet événement par la reconstruction spatiale de quelques objets disparates. C'est par l'introduction du référent photographique, par son usage au sein de l'exposition et par les liens qu'il établissait avec les œuvres et reconstructions exposées, que le LACMA s'écartait de la voie de la reconstruction fidèle au profit d'une lecture du passé actualisée par le moment présent. 
Bien sûr, ce choix a été en partie déterminé par les contraintes pragmatiques du déroulement de l'exposition ${ }^{11}$. Si elle constituait la première rétrospective majeure dédiée par un musée américain à l'art d'avant-garde en Russie, l'exposition du LACMA n'était pas organisée en collaboration avec les musées et autorités soviétiques, les 450 objets exposés (peintures, sculptures, gravures, dessins, photographies, livres, vêtements) provenant de collections publiques et privées de l'Europe occidentale, des Amériques et de l'Australie. (Dans ce sens, The Avant-Garde in Russia différait de l'exposition-manifeste de l'année précédente au Centre Pompidou, Paris-Moscou (1979), marquée par des conflits entre les pays organisateurs ${ }^{12}$.) Les commissaires Stephanie Barron et Maurice Tuchman revendiquaient cette absence de prêts soviétiques comme signe de liberté d'opinion et d'interprétation, mais c'était également un obstacle à toute quête de reconstitution à l'identique. En général, le parti pris de l'exposition du LACMA semble avoir été moins l'exhaustivité que la constitution d'un événement médiatique et culturel à facettes multiples: programmation de films soviétiques et de documentaires conçus pour l'occasion, colloque, conférences «agit-prop » hors les murs, concerts de musique classique russe et soviétique. Outre la mission didactique que déployait ainsi le musée, The Avant-Garde in Russia se démarquait par la volonté d'étendre la notion même d'œuvre au-delà des limites des genres et disciplines artistiques traditionnels. Dans ce but, le LACMA avait recréé l'opéra futuriste Victoire sur le Soleil de $1913^{13}$. D'autres reconstitutions eurent lieu au sein

11. Je tiens à remercier Megan Harley du département d'art moderne du LACMA, Breanne Sallee du département des services photographiques, et particulièrement Douglas Cordell, libraire, pour leur aide dans la consultation des archives de cette exposition. The Avant-Garde in Russia: New Perspectives 1910-1930 a été organisée par les conservateurs Stephanie Barron et Maurice Tuchman. Elle a eu lieu au LACMA du 8 juillet au 28 septembre 1980, puis du 20 novembre 1980 au 15 février 1981, au Hirschhorn Museum and Sculpture Garde de Washington. Toutes les informations factuelles relatives à cette exposition proviennent des communiqués de presse émis par le LACMA tout au long de l'événement et consultés dans les archives du musée. Voir «Opening Press Release - July/ August/September 1980", "Closing Press Release, 9/3/1980».

12. Paris-Moscou s'est tenue au Centre Pompidou-Musée national d'art moderne du 31 janvier au 5 novembre 1979, dans la lignée des expositions inaugurales Paris-New York (1977) et Paris-Berlin (1978). Elle a été suivie, à l'automne 1981, par Moscou-Paris tenue au Musée Pouchkine de Moscou. Pour plus de détails et informations, voir Bernadette Dufrêne (dir.), Centre Pompidou, trente ans d'histoire, Paris, Éditions du Centre Pompidou, 2007.

13. Selon le Los Angeles Times du 9 septembre 1981, les costumes de Victoire sur le Soleil furent recréés par Martha Ferrara d'après les dessins et esquisses de Kazimir 
même de l'espace d'exposition: les décors et costumes constructivistes conçus par Varvara Stepanova en 1922 pour le spectacle de Vsevolod Meyerhold La mort de Tarelkine étaient présentés à même l'entrée, dans un arrangement qui citait la documentation photographique du spectacle tout en remplaçant les comédiens par des poupées grandeur nature; le Modèle du Monument à la III Internationale (1918-1919) de Vladimir Tatline, aujourd'hui disparu, était exposé sous forme de petite maquette dont l'éclairage très théâtral amplifiait l'ombre fantomatique; des modèles de vêtements constructivistes avaient également été recréés, tout comme certaines constructions de l'Obmokhou ou encore des maquettes architecturales ${ }^{14}$. Cette insistance sur l'éclatement de l'objet artistique unitaire, sur la rencontre entre différents arts et sur celle des pratiques artistiques avec la culture matérielle contemporaine, relevait d'une conception de l'histoire mue moins par le désir de monumentaliser le passé que par celui d'établir des ponts avec le présent. Elle constituait autant un hommage à l'expérimentation qu'un lien avec l'actualité des années 1970 et 1980.

En revenant à la présentation de l'exposition de l'Obmokhou et à celle de o,10, dans le cadre de The Avant-Garde in Russia, on peut maintenant suggérer que la réplique-riposte propose une lecture de l'histoire «à rebrousse-poil», en

Malevitch. Tout comme en 1913, les exécutants n'étaient pas des professionnels, mais quatorze étudiants et membres de la faculté du CalArts. Le pianiste Jerry Frohmader «assembled and composed the score based on "extant fragments" of Matiushin's original ». Sur le projet initial, voir La victoire sur le Soleil, Opéra futuriste russe, de Alexeï Kroutchonykh et Mikhaïl Matiouchine. Décors et costumes de Kazimir Malevitch, prologue de Vélimir Khlebnikov. Édition bilingue. Traduction, notes et postface de Valentine et Jean-Claude Marcadé, Lausanne, L'Âge d'Homme, 1976.

14. En soi, la reconstitution d'objets disparus - détruits ou perdus - n'est pas un procédé inhabituel au sein des musées, ni même en relation à l'art du $20^{\circ}$ siècle. Comme le souligne Nathalie Leleu, plusieurs œuvres du "palmarès» de l'art moderne «se distinguent par leur nature sinon par leur fonction: ce sont des répliques, des copies ou des reconstitutions en référence à une œuvre originale qui $[\ldots]$ a été altérée, perdue ou détruite» (Nathalie Leleu, «"Mettre le regard sous le contrôle du toucher". Répliques, copies et reconstitutions au $20^{\circ}$ siècle: les tentations de l'historien de l'art », Les Cahiers du musée national d'art moderne, $\mathrm{n}^{\circ} 93$, automne 2005, p. 84-103, citation p. 84). On peut, suivant Leleu, citer ici non seulement le Modèle de Monument à la III Internationale de Tatline (qui fut l'objet de différentes reconstructions à Stockholm en 1969, à Londres en 1971, à Moscou en 1975 et en 1993, à Paris en 1979), mais aussi les architectones de Malevitch, la Mariée mise à nu par ses célibataires, même de Marcel Duchamp (1915-1923), le Merzbau de Kurt Schwitters (1923-1943), les Demonstrationraüme de Dresde et Hanovre d'El Lissitzky $(1926,1928)$. 
empruntant ce concept critique formulé par Walter Benjamin ${ }^{15}$. Au contraire de la reprise, la riposte insiste sur l'écart entre présent et passé, sur l'impossibilité de revivre aujourd'hui l'expérience passée de l'œuvre. Il est donc possible de voir dans les reconstructions du LACMA une mise à distance délibérée, une «mise en musée » soulignée par la présence de socles et vitrines, partielle et fragmentaire, visant une traduction du passé dans les conditions actuelles et selon les moyens disponibles.

Dans cette conception de l'histoire, le passé n'est pas seulement tenu à l'écart du présent, il rétroagit sur lui. Cela est particulièrement évident dans le dispositif scénographique conçu par Frank Gehry pour The Avant-Garde in Russia. L'agencement sériel et épuré des lattes de bois, exposant le matériau à l'état brut, constitue une citation directe des principes directeurs des décors emblématiques des spectacles constructivistes qui est pleinement assumée par Gehry ${ }^{16}$. Mais il évoque en même temps les pratiques des artistes américains d'allégeance minimaliste et conceptuelle. Se trouve ainsi fondée, à même l'espace d'exposition, la filiation entre l'art d'avant-garde américain des années 1960-1970 et celui de l'avant-garde russe, qui lui est antérieur de cinquante ans. Lun des commissaires, Maurice Tuchman, confirme que ce rapprochement était à l'origine du projet:

The striking formal similarities between the Russians' advanced art of the 1910s and the 1920 and contemporary art, especially in America, prompted us originally to propose an exhibition that would compare the two eras in a single exhibition and

15. Benjamin y voyait la marque d'une résistance face à la capacité assimilatrice de la culture capitaliste: «[La] barbarie inhérente aux biens culturels affecte également le processus par lequel ils ont été transmis de main en main. C'est pourquoi l'historien matérialiste s'écarte autant que possible de ce mouvement de transmission. Il se donne pour tâche de brosser l'histoire à rebrousse-poil. » Walter Benjamin, «Sur le concept d'histoire», Euvres, tome III, trad. Maurice de Gandillac, Rainer Rochlitz et Pierre Rusch, Paris, Gallimard, coll. «Folio. Essais», 2000, p. 433.

16. Cette résonance entre scénographie et œuvres exposées semble avoir régi les collaborations de Frank Gehry avec le LACMA, et ce projet en particulier: «Working closely with the museum's curatorial staff [...] the goal was always to create environments compatible with the works displayed without competing for the visitor's gaze. [...] Gehry's installation recalled constructivist stage techniques by exposing studs spaced at various intervals; color dominant in the paintings and graphics of the period - reds, blacks, whites, and grays - were used on the partitions to define display groupings. » Francesco Dal Co and Kurt W. Forster (dir.), Frank O. Gehry. The Complete Works, New York, The Monacelli Press, 1998, p. 216. 
catalog. Ultimately, however, we decided upon a fuller presentation of Russian art, utilizing the opportunity to show Suprematism and Constructivism to the utmost ${ }^{17}$.

Selon une autre version due à la co-commissaire Stephanie Barron, le retrait du volet contemporain de l'exposition était plutôt une décision politique justifiée par l'ultimatum posé par l'organisme subventionnaire (National Endowment for Arts and Humanities) dans le contexte de la guerre froide ${ }^{18}$. Même en l'absence d'œuvres contemporaines, la similarité entre le dispositif de l'exposition et les pratiques artistiques récentes trouve écho dans la réception journalistique de l'exposition, donnant lieu à des critiques parfois acerbes ${ }^{19}$.

Or, si les reconstructions et le dispositif scénographique au sein de The Avant-Garde in Russia se donnent à voir comme traductions, voire transferts, de l'expérience passée, créant un lien avec l'expérience de l'art américain contemporain, l'exposition donnait également à voir un autre usage de la photographie. La surabondance de photographies documentaires, souvent agrandies plus que nature, favorisait au contraire l'illusion d'une expérience immersive, participative du spectateur à l'histoire. Photographies des fêtes publiques révolutionnaires ou photogrammes de films de propagande, les agrandissements photographiques à l'échelle du visiteur étaient placés sur les murs de façon à scander le parcours du spectateur, à guider son corps à travers les espaces de l'exposition, à cadencer ses pas au rythme des foules révolutionnaires en marche. Les propos d'une journaliste soulignent cet effet théâtral des photographies documentaires, pour la plupart issues des archives privées de l'historien de l'art polonais Szymon Bojko: «[...] the exhibition's huge, blown-up photographs of mass theater spectacles and

17. Stephanie Barron et Maurice Tuchman (dir.), The Avant-Garde in Russia, 19101930: New Perspectives, Exhibition, Los Angeles County Museum of Art (L.A.C.M.A.), 8 juillet-28 septembre 1980, p. 118. Les filiations que brosse l'article de Tuchman «The Russian Avant-Garde and the Contemporary Artist», p. 118-121, reposent sur des entretiens avec artistes et critiques d'art contemporains: Carl Andre, Mel Bochner, Bruce Boice, Mark di Suvero, Jeremy Gilbert-Rolfe, Donald Judd, Sol LeWitt, Loren Madsen, Robert Morris, Brian O’Doherty (Patrick Ireland), Richard Serra et Frank Stella; il considère également l'œuvre d'autres artistes décédés comme Ad Reinhardt ou Jean Tinguely.

18. Propos rapportés par Manuela Hoelterhoff dans son article «Art: The Revolution that Failed», Wall Street Journal (Pacific Ed.), 20 juin 1980.

19. Voir Joan Hugo, "The Mission of the Avant-Garde », Artweek, Oakland, 16 août 1980: «For what emerges from the installation is a stylish, cool arrangement of aesthetic objects, organized without real focus [...] chosen more for their relationship to American work of the past twenty years than for their relationship to ideas or to each other. » 
portraits of intense-looking, mostly young artists [...] lend a sense of drama to the installation ${ }^{20}$.»

The Avant-Garde in Russia semble ainsi avoir déployé au moins deux discours sur l'histoire au moyen de la photographie: le recours à ces vues documentaires favorisant plutôt une fétichisation de l'histoire, contrairement à la posture ironique et à l'insistance sur l'inachevé que conviaient les couplages entre référent photographique et reconstruction tridimensionnelle, dans le cas de o,10 ou de l'exposition de l'Obmokhou.

Il est difficile de terminer cette brève analyse sans relier cette omniprésence de la photographie au sein de cette exposition au discours élaboré dans les mêmes années par les critiques d'art américains comme Douglas Crimp sur l'«activité photographique»du postmodernisme. Dans son article éponyme, publié à l'hiver 1980 dans October, Crimp relie la crise du modèle moderniste du musée d'art à l'apparition de nouvelles pratiques artistiques qui tentent de «récupérer l'auratique » : la résurrection de la peinture expressionniste et l'affirmation de «la photographie en tant qu'art». Pour Crimp, la force des artistes travaillant dans la dernière veine (Sherrie Levine, Cindy Sherman, Richard Prince - dont il avait présenté pour la première fois le travail en 1977 dans l'exposition Pictures) consiste précisément en la démonstration du statut toujours déjà reproductible, toujours déjà vu, de l'image photographique qui trace une croix sur les prétentions à l'originalité de l'art moderniste ${ }^{21}$. Parmi les compte-rendus critiques de The Avant-Garde in Russia, au moins un journaliste semble partager cette posture postmoderniste qui donne prééminence au relais photographique sur l'expérience directe de l'œuvre que l'auteur juge inaccessible à cause de l'effondrement des utopies qui l'avaient soutenue:

The photograph of Malevich's 1915 exhibition conveys a greater sense of vitality than most of his actual paintings [...]. It may be that art perishes when one begins to separate the idea from the material (or when material becomes the only idea), spirit from body. In any event, where the exhibition comes alive it is principally as living history, only in the rarest cases as living art ${ }^{22}$.

20. Hoelterhoff, 1980.

21. Douglas Crimp, «The Photographic Activity of Postmodernism », October, n ${ }^{\circ}$ 15, hiver 1980, p. 91-101.

22. Thomas Albright, "A Staggering Russian Avant-Garde Show», San Francisco Chronicle and Examiner, 20 juillet 1980. 


\section{LE MUSÉE IRONIQUE}

Un deuxième exemple de «riposte », beaucoup plus récent, apporte une stratification additionnelle aux liens entre la reproduction photographique et son pendant, la reconstruction tridimensionnelle d'expositions passées. Il s'agit du projet Nachbau réalisé en 2007 par l'artiste Simon Starling en collaboration avec le musée Folkwang d'Essen, en Allemagne, au moment même où celui-ci procédait à d'importants travaux de rénovation architecturale.

Simon Starling développe depuis le milieu des années 1990 un travail in situ de filiation néoconceptuelle qui prend ancrage dans le contexte et dans l'histoire élargie - tant artistique qu'industrielle, économique ou politique - du lieu d'exposition. Ses productions ne se limitent pas à des interventions éphémères comme celles des artistes des générations précédentes, tel Michael Asher, mais donnent aussi lieu à des objets, souvent de faux ready-made qui survivent à l'exposition. En général accompagnées d'un livre d'artiste qui agit comme complément au projet physique, les œuvres de Starling proposent des récits à mi-chemin entre la « référence » historique et la «fiction» artistique $^{23}$.

Le projet Nachbau, comme tout le travail de Starling, convoque l'histoire du lieu où son intervention prend place et forme - le Museum Folkwang d'Essen - et tient compte des spécificités du moment d'exécution de l'installation : la démolition imminente d'un bâtiment du musée construit en 1983 en vue du réaménagement du site muséal par le bureau de l'architecte David Chipperfield ${ }^{24}$.

Avant de commenter Nachbau, il importe de présenter la spécificité du Museum Folkwang d'Essen. L'acte fondateur de son histoire est l'acquisition, en 1921, des collections d'un des premiers musées européens d'art moderne, fondé en 1902 par Karl Ernst Osthaus dans sa ville natale de Hagen. Héritier de riches industriels, Osthaus avait investi sa fortune dans le projet d'un musée quasi anthropologique, réunissant objets issus des sciences naturelles, œuvres d'art moderne (de Cézanne à l'expressionnisme allemand) et objets d'art cérémoniels

23. Voir Simon Starling, Thereherethenthere, MAC-VAL - Parc Saint-Léger, 2010, publication bilingue anglais-français, 2 volumes.

24. Simon Starling, Nachbau (catalogue d'exposition, Museum Folkwang, Essen, 20.04-01.07 2007), Essen, Museum Folkwang, 2 volumes, 2007, p. 7. Voir la section «Architecture» du site de présentation du Museum Folkwang www.museum-folkwang. de/en/museum-folkwang/architecture.html (dernière consultation le zo juillet 2010) pour plus de détails sur le projet de David Chipperfield. Les nouveaux bâtiments du musée furent inaugurés en 2010. 


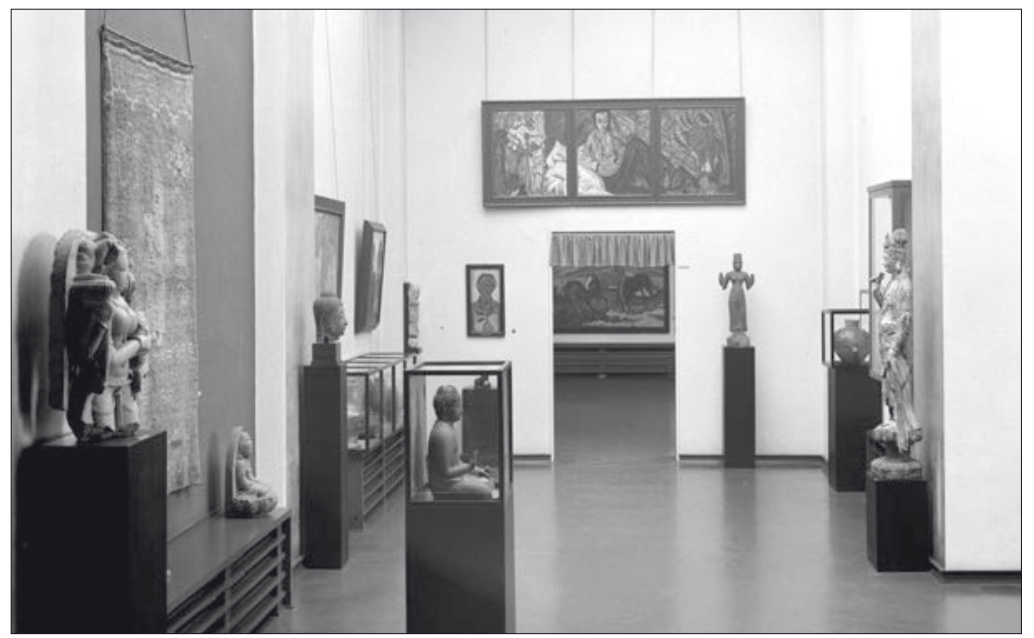

Fig. 5: Albert Renger-Patzsch, Vue intérieure d'une salle du Museum Folkwang à Essen, ca. 1930, accrochage d'Erich Gosebruch. Épreuve au gélatino-bromure d'argent, 16,8 x 22,7 cm. (C) La collection du Museum Folkwang, Essen.

ou quotidiens issus de cultures non européennes. À la mort d'Osthaus en 1921, la ville de Hagen, hostile à son initiative, refuse de prendre le musée en charge. La collection Osthaus trouve alors preneur dans la ville d'Essen qui fonde le musée Folkwang en 1922, en fusionnant sa nouvelle acquisition avec le musée municipal existant. La collection Osthaus y sera exposée à partir de 1929 dans un bâtiment construit à cette occasion par l'architecte Eduard Körner. Célébré par de nombreuses personnalités de la scène artistique internationale, le Museum Folkwang avait notamment impressionné le futur directeur du Museum of Modern Art, Alfred H. Barr, et son architecte, Philip Johnson ${ }^{25}$. Pendant la Seconde Guerre mondiale, tous les bâtiments du musée ont été démolis par des bombardements. Quant aux œuvres de la collection, environ 1400 furent classées «dégénérées » et confisquées par le pouvoir national-socialiste. Plusieurs furent exposées à l'Exposition d'art dégénéré à Munich de 1937. À partir des années 1950, certaines ont pu être rachetées ou récupérées, d’autres ont disparu ou se trouvent dispersées dans d'autres collections ${ }^{26}$.

25. Voir Mary Anne Staniszewski, The Power of Display. A History of Exhibition Installations at the Museum of Modern Art, Cambridge (Mass.) et Londres, MIT Press, 1998.

26. Pour les informations factuelles sur l'histoire du musée Folkwang à Essen, voir Museum Folkwang, «Pressemitteilung: Simon Starling-Nachbau», Zeitgenössische Kunst im Museum Folkwang. Eine Kooperation mit der RWE AG, 20 avril-1 ${ }^{\text {er }}$ juillet 2007; Michael Fehr, «A Museum and Its Memory. The Art of Recovering History,» dans 


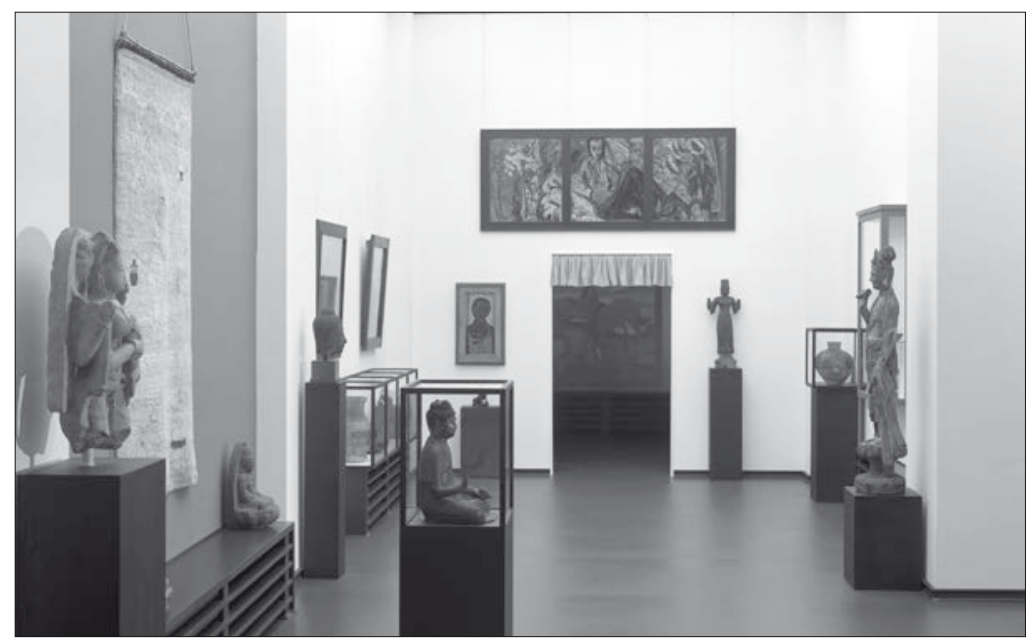

Fig. 6: Simon Starling, Nachbau (Reconstruction), une épreuve au gélatino-bromure d'argent, 17,3 × $23 \mathrm{~cm}$. (c) Simon Starling.

Le projet Nachbau est inspiré d'une série de photographies réalisées dans les années 1930 par le photographe allemand Albert Renger-Patzsch, principalement connu comme l'auteur du livre de photographies Die Welt ist schön (Le monde est beau, 1928). Entre 1929 et 1944, en échange d'un espace d'atelier au sein du Museum Folkwang d'Essen, Renger-Patzsch a agi à titre de photographe non officiel de ses collections, documentant aussi bien des œuvres et objets individuels que des vitrines ou des vues de salles entières. Dans sa description du projet, Simon Starling précise que cette entente n'était pas formellement reconnue par l'institution muséale, contribuant à ce que les archives photographiques demeurent longtemps inconnues au sein de la succession de Renger-Patzsch ${ }^{27}$. Pour Nachbau, Starling choisit quatre des vues

Susan A. Crane (dir.), Museums and Memory, Stanford, Stanford University Press, 2000, p. 35-59, ainsi que la section «Chronicle» du site Internet du Museum Folkwang à Essen www.museum-folkwang.de/en/museum-folkwang/chronik.html (dernière consultation le 20 juillet 2010).

27. Elles font actuellement partie du département de photographies du musée d'Essen. Voir «Pressemitteilung: Simon Starling-Nachbau», Zeitgenössische Kunst im Museum Folkwang. Eine Kooperation mit der RWE AG, 20 avril-1-1 juillet 2007; Simon Starling, «Replication: Some Thoughts, Some Works», Tate Papers, n 8, automne 2007, disponible à www.tate.org.uk/research/tateresearch/tatepapers/opautumn/starling.htm (dernière consultation le 20 juillet 2010). Voir aussi la présentation du travail de Renger-Patzsch dans Starling, Nachbau, 2007, vol. II, p. 63. 
photographiques de Renger-Patzsch qui, ensemble, représentent presque intégralement l'une des salles d'exposition (fig. 5).

La réplique réalisée par Nachbau est tripartite. D’une part, Starling a reconstruit, grandeur nature, une salle du bâtiment désormais inexistant de Körner (Altbau) d'après les photographies de Renger-Patzsch. Larchitecture et l'aménagement intérieurs de la salle sont reproduits à l'identique par l'ajout de faux murs et de corniches, et par l'introduction de mobilier de l'époque. D'autre part, Starling a réitéré l'accrochage conçu et mis en place dès 1929 par le directeur du musée et ami d'Osthaus, Ernst Gosebruch. Conformément à l'attitude anthropologique de Karl Ernst Osthaus, la présentation de cette salle juxtaposait œuvres d'art modernes (tableaux de Giorgio De Chirico, Erich Heckel, Franz Marc, Paula Modersohn-Becker, Otto Müller, Emil Nolde, sculpture d'Aristide Maillol) et objets cultuels ou utilitaires de cultures non occidentales (plusieurs vases et figurines de céramique chinois, un tissu tibétain de la fin du $18^{\text {e }}$ siècle, des sculptures de Bouddha provenant du Japon et de la Chine et couvrant un laps de temps assez vaste, du $12^{\mathrm{e}}$ au début du $20^{\mathrm{e}}$ siècle). Ce type d'accrochage faisait ressortir non seulement les techniques d'inspiration primitiviste caractéristiques de l'art moderne, mais, plus généralement, inscrivait les productions artistiques européennes dans une temporalité historique plus vaste. Deux éléments distinguaient l'accrochage de 2007 de celui de 1929: l'absence d'une figure bouddhique de Chine visible sur le mur de gauche dans la photographie de Renger-Patzsch, et la présentation des tableaux de Marc, Mueller, Heckel et De Chirico sous forme de reproductions couleur, les originaux n'appartenant plus au Museum Folkwang d'Essen. Enfin, dans un troisième temps, Starling a refait la documentation photographique de l'installation en réitérant les mêmes cadrages et points de vue que RengerPatzsch (même si l'on peut remarquer chez Starling une finition plus soignée, peut-être due à une optique plus perfectionnée qui, combinée avec certains effets d'éclairage, fait ressortir davantage la volumétrie des corps et les détails dans les œuvres) (fig. 6).

On comprend alors la polysémie de l'intitulé Nachbau. À première vue, il offre un jeu de mots sur le lieu de sa tenue: «après-construction ", Nachbau prend place dans une des ailes du musée d'Essen au moment même où celleci devait être démolie pour laisser place au Neubau de David Chipperfield. Mais Nachbau est aussi le terme utilisé en allemand pour désigner la construction d'après un modèle déposé, la reproduction, de même qu'un ajout de construction. En l'occurrence, les «modèles déposés » d'après lesquels il s'agit ici de re-construire (nach-bauen) sont des spectres: une collection démantelée 
et dispersée, un accrochage temporaire, un bâtiment démoli, une expérience passée.

Linstallation a eu lieu au sein du musée Folkwang du 20 avril au $1^{\text {er }}$ juillet 2007. Une fois exécutée et photographiée, elle a été démantelée, tout comme le bâtiment où elle était présentée. Dans une démarche habituelle pour Starling, le projet a généré une publication en deux volumes presque identiques - l'un en allemand, l'autre en anglais - qui jumellent les fonctions de catalogue et d'œuvre ${ }^{28}$. Si tous deux partagent la même mise en page, appareil critique et article théorique (de Bruno Haas), l'édition allemande comprend un luxueux dossier photographique de toutes les vues prises par Renger-Patzsch dans les collections du musée d'Essen durant son séjour, alors que l'édition anglaise ne comporte que les quatre vues prises dans la salle reconstituée par Starling. Elles sont placées aux mêmes endroits que les vues équivalentes de Renger-Patzsch, les autres pages étant laissées vides. En plus de cette publication, l'installation a donné lieu à quatre photographies en noir et blanc encadrées sous passe-partout qui se présentent comme une œuvre à part entière ${ }^{29}$.

Contrairement à d'autres reconstitutions, ce projet a donc pour but ultime moins l'installation que la production de sa photographie. En d'autres termes, la reconstruction spatiale n'est qu'une étape, le véritable objectif consistant en la reconstruction photographique. Ce constat nous permet de revenir à la question qui sous-tend cette enquête sur la reconstitution d'expositions: quel est le statut de ce double photographique? À quelle expérience convie-t-il ses spectateurs?

Les photographies de Starling se placent en dialogue avec le travail appropriationniste d'artistes comme Sherrie Levine qui, au tournant des années 1980, avait re-photographié des images emblématiques d'Edward Weston et Walker Evans en posant à vif la question de l'auteur et de la valeur d'originalité comme concepts fondamentaux de l'histoire de l'art moderniste ${ }^{30}$. Toutefois, la démarche adoptée par Starling introduit une différence de taille. Plutôt que de reproduire mécaniquement des photographies déjà existantes, ses photographies jouent sur l'écart entre des images presque parfaitement ressemblantes et des expériences

28. Starling, 2007. Les projets de Starling sont généralement accompagnés de publications très soigneusement conçues. Il exploite à plusieurs reprises le format du coffret en deux volumes en jouant sur la limite entre catalogue et livre d'artiste, y compris dans le cadre de sa récente rétrospective en France (Starling, 2010).

29. Il s'agit d'une œuvre multiple. La Kadist Art Foundation de Paris en possède un exemplaire (quatre tirages argentiques encadrés, $72,8 \times 64,8 \times 4,5 \mathrm{~cm}$ chaque photographie encadrée).

30. Voir Crimp, 1980, pour une première élaboration théorique. 
temporelles et historiques qui ne peuvent coïncider (à la différence de Levine, cet écart imperceptible nécessite un travail de reconstitution et non seulement de reproduction mécanique). Une manière d'interpréter ce travail de reconstruction photographique consiste à admettre qu'en plus d'accepter l'écart temporel entre le moment passé et le moment de sa réitération présente, la nouvelle prise de vue du projet Nachbau produit l'image de cet écart - qui est en même temps l'image de la ressemblance.

Sur le plan institutionnel également, Nachbau pose un défi au Museum Folkwang d'Essen: ni le jeu des quatre photographies ni la reconstruction ne demeurent dans la collection permanente du musée. À la différence de la salle Obmokhou du musée Tretiakov, le Museum Folkwang ne fait pas de la mise en exposition de Gosebruch une «installation » permanente évoquant une époque passée ou un accrochage emblématique. Quant au reste photographique qui «survit» au projet de Starling, à la différence des blow-ups du LACMA, il ne dessert pas l'objectif de transporter le visiteur, par un effet d'immersion à l'image, dans un passé fantasmé. Encadrées sous passe-partout, les quatre vues reconstituées se donnent désormais comme œuvres d'art à propos du musée, œuvres qui exposent le musée s'exposant à sa propre histoire.

Cette attitude, l'historien de l'art Stephen Bann l'avait qualifiée de «musée ironique ", désignant ainsi un rapport à l'histoire succédant à la fois au modèle de la métonymie (le fragment pour le tout) ou de la synecdoque (la reconstruction de type period room). Le «musée ironique» repose sur la reconnaissance à la fois du caractère fictionnel du musée et de son potentiel poétique ${ }^{31}$. Le musée d'Essen n'est pas le premier à adopter une telle posture. Il a au moins un précédent, également issu du projet muséographique de Karl-Ernst Osthaus. Il s'agit du Museum Folkwang de Hagen, celui même qu'Osthaus avait fondé en 1902. Quelque 90 ans plus tard, sous la direction de Michael Fehr, le musée de Hagen passait une commande pour sa collection permanente à un artiste anonyme actif à New York, se présentant comme Salon de Fleurus. Intitulée Moderne Kunst from the Museum Folkwang, Hagen, 1904, l'installation de peintures qui résulta de cette commande reproduisait, sous forme de copies peintes, plusieurs tableaux de la collection Osthaus qui n’appartenaient plus au musée de Hagen ainsi que

31. Stephen Bann, «Poetics of the Museum: Lenoir and Du Sommerard», The Clothing of Clio: A Study of the Representation of History in Nineteenth-Century Britain and France, Cambridge, Cambridge University Press, 1984, p. 77-92. 
plusieurs vues de l'accrochage d'époque ${ }^{32}$. Les tableaux étaient exécutés d'après des photographies du musée de Karl-Ernst Osthaus de 1905. Encore une fois, le contexte d'exposition se trouvait intégré comme partie prenante de l'histoire des œuvres.

Cette installation, qui devait en quelque sorte réintroduire la mémoire de la collection absente, s'insérait dans une série de projets par lesquels Michael Fehr avait tenté de penser le musée de Hagen non pas, comme de coutume, à partir de ses collections ou possessions mais bien au contraire, à partir de ses manques et oublis ${ }^{33}$. À la différence du Nachbau de Starling, et une quinzaine d'années avant son projet, la source photographique n'avait pas donné lieu ici à un tirage précieusement encadré sous passe-partout, mais à une série de (mauvaises) peintures. Certes, à sa façon, chacun de ces projets augmente la valeur ajoutée du musée qui les accueille ou qui en passe commande. Mais ils diminuent également la valeur ajoutée des reconstructions d'exposition, en insistant davantage, par les transferts intermédiatiques à l'œuvre, sur les écarts, failles et oublis dans la quête de l'expérience passée.

32. Ce projet est reproduit dans Inke Arns et Walter Benjamin (dir.), What is Modern Art? Group Show, vol. 1, Berlin, Künstlerhaus Bethanien, 2006, p. 20-23.

33. Sur le travail de Michael Fehr au sein du Musée Karl-Ernst Osthaus et sa conception du musée en général, voir Fehr, 2000. 